\title{
Evaluación de anemia post intervención nutricional en niños de comunidades rurales de Caazapá, Paraguay
}

\author{
Evaluation of anemia after nutritional interventions in \\ children living in rural communities in Caazapa, Paraguay
}

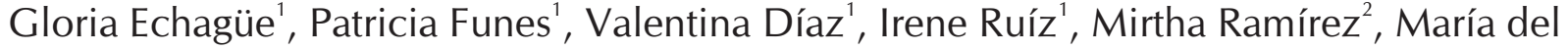 \\ Carmen Franco ${ }^{2}$, Liliana Sosa ${ }^{1}$
}

\section{RESUMEN}

Introducción: Se ha descrito la heterogeneidad del impacto de la anemia según el área geográfica; en el sector rural se ve agravado afectando a la población más vulnerable. Objetivo: evaluar el efecto del suplemento nutricional en niños anémicos menores de cinco años indígenas y no indígenas, en comunidades rurales del Departamento de Caazapá. Materiales y métodos: Estudio observacional transversal. Fueron estudiados una cohorte de 109 niños menores de cinco años indígenas y no indígenas cuyos padres brindaron su consentimiento informado, a quienes se les suministró sulfato ferroso según edad y peso. Resultados: Se encontró un aumento significativo de las medianas de hemoglobina, después de la intervención, de 5,0 g/L en el grupo anémico de indígenas y en los no indígenas de 6,0 g/L; el 31\% de los niños indígenas y el $84 \%$ de los no indígenas dejaron de ser anémicos, post intervención. Conclusión: Ambas poblaciones de niños anémicos indígenas y no indígenas presentaron un aumento significativo de los niveles medios de hemoglobina post tratamiento con suplemento con hierro y una mayoría presentó una mejoría a un estado no anémico o del grado de la anemia.

Palabras claves: anemia, hierro, uso terapéutico, población vulnerable.

\section{INTRODUCCIÓN}

La anemia es considerada por la Organización Mundial de la Salud un problema de salud pública ${ }^{(1)}$, e interfiere con el aprendizaje, el desarrollo cognitivo

\section{ABSTRACT}

Introduction: The heterogeneity of the impact of anemia has been described according to geographic area; in rural areas the impact is greater, affecting the most vulnerable populations. Objective: to evaluate the effect of nutritional supplementation in anemic children, both indigenous and non-indigenous, under five years of age, in rural communities in the Department of Caazapá. Materials and methods: This was a cross-sectional, observational study. We studied a cohort of 109 indigenous and non-indigenous children under five years of age whose parents gave their informed consent and were given ferrous sulfate based on age and weight. Results: There was a significant increase in hemoglobin medians, after the intervention, of $5.0 \mathrm{~g} / \mathrm{L}$ in the anemic group of indigenous children and of $6.0 \mathrm{~g} / \mathrm{L}$ in the nonindigenous children; $31 \%$ of indigenous children and $84 \%$ of non-indigenous children ceased to be anemic after the intervention. Conclusion: Both the indigenous and nonindigenous populations of anemic children showed a significant increase in their average levels of hemoglobin after iron supplementation and a majority improved to a milder degree of anemia or to a resolution of their anemia.

Keywords: Anemia, iron, therapeutic use, vulnerable population

\footnotetext{
${ }^{1}$ Universidad Nacional de Asunción. Instituto de Investigaciones en Ciencias de la Salud. San Lorenzo, Paraguay.

${ }^{2}$ Fundación Acción Contra el Hambre. Asunción, Paraguay.

Correspondencia: Liliana Sosa. Correo: aclinicos@iics.una.py

Conflicto de interés: Los autores declaran no poseer conflicto de interés

Recibido: 6/03/2019 Aceptado: 22/07/2019

DOI: https://doi.org/10.31698/ped.46022019006

Este es un artículo publicado en acceso abierto bajo una Licencia Creative Commons CC-BY 4.0
} 
Paraguay la nutrición se encuentra relacionada a la condición de pobreza y el acceso limitado a servicios sociales básicos y los grupos vulnerables, que viven en territorios con menor nivel de inversión pública ${ }^{(4)}$. Aunque América Latina ha tenido avances en los ámbitos social y económico en los últimos años, grupos poblacionales aún viven en situación de inseguridad alimentaria y pobreza, los cuales se ven agravados en el sector rural afectando a la población más vulnerable, niños, mujeres e indígenas ${ }^{(5)}$.

Intervenciones nutricionales que se llevan a cabo antes de los dos años son cruciales para asegurar la productividad, el nivel académico y social, pues casi todo el retardo en el crecimiento se presenta durante este periodo ${ }^{(6)}$. Los programas de suplementación han sido eficaces en condiciones controladas sin embargo, existen barreras técnicas y prácticas: información limitada sobre la efectividad de las intervenciones de suplementación, los efectos secundarios, la baja adherencia y las limitaciones de oferta / distribución. En algunos países, la ubicación geográfica, las variaciones en el idioma y el tamaño de la población pueden hacer que el costo de los programas de intervención a través de programas de información, educación y comunicación sea muy alto $^{(7)}$.Otro limitante es la baja priorización del problema de la anemia al ser una condición generalmente asintomática, donde los padres y las autoridades de salud no logran sensibilizarse sobre el impacto y las consecuencias en los niños ${ }^{(8)}$.

Aparco y Huamán citan que no existe un suplemento mágico que resuelva los problemas de acceso económico, geográfico o cultural a los servicios de salud, las dificultades de la madre en el uso apropiado y el consumo adecuado del suplemento en sus hijos, el sistema de entrega del suplemento, motivación del personal de salud, entre otros ${ }^{(9)}$.

En Paraguay, el programa PANI, Programa Alimentario Nutricional Integral del Ministerio de Salud Pública y Bienestar Social - MSP y BS, es el responsable de la prevención, asistencia y control de la desnutrición de niños menores de cinco años de edad y mujeres embarazadas que se encuentren en situación de pobreza, bajo peso o cualquier situación de vulnerabilidad nutricional, a través de la atención sanitaria en los puestos de salud y la entrega del complemento alimentario, consistente en leche enriquecida con hierro, zinc, cobre y vitamina $C$, sin embargo, sufre inconvenientes tales como la falta de disponibilidad presupuestaria o de recursos humanos ${ }^{(10)}$, que hace difícil llegar a las comunidades potenciales o en riesgo. En el estudio realizado en el Departamento de Concepción, Paraguay, en niños menores de 5 años, beneficiarios del programa alimentario PANI que acudían al Hospital de Concepción, se constató una mejoría significativa de los parámetros antropométricos y bioquímicos ${ }^{(11)}$. La OMS sugiere considerar la suplementación como una medida preventiva de salud pública para controlar la deficiencia de hierro en poblaciones con alto riesgo de deficiencia de hierro y anemia, previo estudio de factibilidad y posibles limitaciones en su aplicación ${ }^{(12)}$.

Un estudio anterior realizado en una cohorte de niños menores de cinco años, de comunidades rurales indígenas y no indígenas en el departamento de Caazapá, encontró una prevalencia de anemia del $74 \%$ en niños indígenas y de $46 \%$ en niños no indígenas ${ }^{(13)}$, teniendo en cuenta ese hallazgo, el objetivo del presente trabajo fue evaluar el efecto de la suplementación con sulfato ferroso a los niños anémicos de dicha cohorte de comunidades rurales del Departamento de Caazapá.

\section{MATERIALES Y METODOS}

\section{Diseño de la investigación}

Estudio observacional transversal, realizado en el año 2013 a una cohorte de 109 niños menores de cinco años indígenas y no indígenas de los Distritos de Abai y Tavai del Departamento de Caazapá, encontrados anémicos siete meses antes. Los niños fueron atendidos por personal médico de los Puestos de Salud del Ministerio de Salud Pública y Bienestar Social cercanos a sus comunidades, quienes proporcionaron a los padres o tutores de los niños en situación de anemia, un suplemento conteniendo hierro con las indicaciones de acuerdo a la edad y peso de cada niño. A los niños de 6 a 59 meses se les administró vía oral $5 \mathrm{mg} / \mathrm{Kg} /$ día de hierro elemental; los de 6 a 11 meses recibieron sulfato ferroso en gotas y los niños de 12 a 59 meses tomaron un jarabe conteniendo sulfato ferroso con vitaminas ${ }^{(14)}$. Concomitantemente recibieron 
tratamiento antiparasitario de acuerdo al protocolo del Ministerio de Salud Pública y Bienestar Social, para vermes: albendazol, 1 ampolla de $400 \mathrm{mg}$ por dia, durante 5 días seguidos, en niños desde los 24 meses hasta los 59 meses, y mebendazol para niños menores de 24 meses, $5 \mathrm{ml}$ por vía oral cada 12 horas, durante tres días. Para protozoarios: metronidazol cuya dosis fue de $20 \mathrm{mg} / \mathrm{kg} /$ día durante 7 días en niños desde los 12 meses hasta los 59 meses.

\section{Etapas del seguimiento de la cohorte:}

Primera etapa (marzo/mayo 2012): análisis de hemoglobina realizado a una cohorte de 226 niños anémicos y no anémicos.

Segunda etapa (diciembre 2012/marzo 2013): intervención con sulfato ferroso a la cohorte de 109 niños encontrados anémicos en la primera etapa

Tercera etapa (febrero/mayo 2013): análisis de hemoglobina realizado a la cohorte de niños anémicos a los dos meses después de iniciado el tratamiento.

Para el análisis de la anemia los niños fueron autoemparejados y asignados en dos grupos:

Grupo 1:77 niños indígenas anémicos

Grupo 2:32 niños no indígenas anémicos

Se consideró como abandono a los niños que interrumpieron definitivamente la ingesta del suplemento y como pérdida cuando no fue posible obtener información de ellos o se negaron a volver a participar en la segunda etapa.

\section{Tamaño de la muestra}

Para la estimación del tamaño de la muestra se utilizaron las diferencias esperadas de las medias de las concentraciones de hemoglobina. Se recurrió a la diferencia reportada entre las evaluaciones basal y final del estudio de Urquidi et al. realizado en Bolivia, en el cual la media basal de hemoglobina fue de $115 \pm 15 \mathrm{~g} / \mathrm{L}$ y la media post intervención de $126 \pm 12 \mathrm{~g} / \mathrm{L}$, con lo cual se obtuvo la diferencia con un incremento de $11 \mathrm{~g} / \mathrm{L}(1,1 \mathrm{~g} / \mathrm{dL})$ de hemoglobina para sulfato ferroso ${ }^{(15)}$. El tamaño de la muestra fue calculado para detectar una diferencia de $7 \mathrm{~g} / \mathrm{L}(0,7$ $\mathrm{g} / \mathrm{dL}$ ) en la media de hemoglobina, entre las evaluaciones basal y post intervención, así sería necesario estudiar como mínimo 26 niños por grupo en cada etapa, considerando un $\alpha$ unilateral de 0,05 y una potencia $\beta$ de 0,20 según la tabla para calcular tamaños de muestra necesario por grupo al usar la prueba de la $t$ para comparar medias de variables contínuas ${ }^{(16)}$.

\section{Criterios de inclusión y exclusión}

Para este estudio fueron incluidos los niños anémicos cuyos padres dieron su consentimiento. Se excluyeron a niños que cursaran con enfermedades agudas (diarrea, tos, fiebre, gripe) desde 15 días antes de la toma de muestra o con presencia de enfermedades neoplásicas.

Las variables estudiadas fueron datos demográficos edad, sexo, peso, talla, estado nutricional y coproparasitológico, presencia de signos y síntomas de enfermedad, cumplimiento del tratamiento. Los datos fueron recogidos por medio de una encuesta con preguntas cerradas a los padres o tutores.

Para la medición de la hemoglobina $(\mathrm{Hb})$ se obtuvo una muestra de sangre venosa del brazo, el procesamiento se realizó según el método de la cianometahemoglobina, en el contador hematológico AVX micros 60. Se definió anemia de acuerdo a la $\mathrm{OMS}^{(17)}$, con una hemoglobina inferior a $110 \mathrm{~g} / \mathrm{L}$ y grados de anemia según: leve 100 a 109 g/L de hemoglobina, moderada 70 a $99 \mathrm{~g} / \mathrm{L}$ y grave menos de $70 \mathrm{~g} / \mathrm{l}$.

Para la medición del peso se utilizó una balanza digital de capacidad de hasta $120 \mathrm{Kg}$, marca Welmy, ajustada a cero antes de cada medición y registrándose en unidades de kilogramos $(\mathrm{kg})$ en los niños mayores de dos años. Los niños menores de 2 años, fueron pesados en la misma balanza, primero con sus madres y luego la madre sola, obteniéndose el pesaje del niño por la diferencia de pesos entre ambos. La talla se obtuvo mediante el empleo de una cinta métrica y se registró en centímetros $(\mathrm{cm})$. En niños menores de dos años fue medida en posición de decúbito dorsal y en niños mayores de dos años, en posición de pie.

Los datos antropométricos fueron evaluados basándose en los nuevos estándares de referencia establecidos por la Organización Mundial de la Salud (OMS) en los Patrones de Crecimiento Infantil 
de $2006^{(18)}$ usando el puntaje Z, el cual es definido por la desviación estándar (DE), para peso según edad $(\mathrm{P} / \mathrm{E})$, en niños menores de 2 años, peso para la talla $(\mathrm{P} / \mathrm{T})$, en niños de 2 hasta 5 años de edad. Los puntos de corte fueron: P/E: desnutrición global grave $(<-$ $3 \mathrm{DE})$, desnutrición global moderada ( $<-2 \mathrm{DE}$ hasta $3 \mathrm{DE})$, riesgo de desnutrición ( $<-1 \mathrm{DE}$ hasta $-2 \mathrm{DE})$, peso adecuado ( $\geq-1 \mathrm{DE}) ; \mathrm{P} / \mathrm{T}$ : desnutrición aguda grave $(<-3 \mathrm{DE})$, desnutrición aguda moderada $(<-2$ DE hasta -3DE), riesgo de desnutrición (<-1 DE hasta $-2 \mathrm{DE})$, con peso adecuado $(\geq-1 \mathrm{DE}$ hasta $+1 \mathrm{DE})$, sobrepeso (>+1 DE hasta + $2 \mathrm{DE})$, obesidad ( $>+2 \mathrm{DE})$. Para el análisis coproparasitológico de las muestras de heces simples, se utilizó el método directo y el de flotación de Willis.

\section{Asuntos Éticos}

Se respetaron los 3 principios de la ética e investigación. Participaron del estudio todos los niños menores de 5 años que contaron con el consentimiento informado de los padres o tutores. Los resultados del estudio fueron socializados con los mismos y a los niños se les brindó asistencia médica. Todos los datos y las muestras biológicas fueron resguardados de acuerdo a un código asignado protegiendo la identidad. El presente estudio fue evaluado y aprobado por el Comité Científico y de Ética del Instituto de Investigaciones en Ciencias de la Salud.

\section{Procesamiento y análisis de datos}

La base de datos fue cargada en planilla Excel 2003 y el análisis se realizó utilizando el paquete estadístico para Windows SPSS.

Se utilizó estadística descriptiva en términos de promedios, desviaciones estándar, medianas, rangos intercuartilícos y proporciones según correspondiera. Para comparar las medianas de datos emparejados no paramétricos se utilizó la prueba de rango de signo de Wilcoxon. Los resultados se consideraron significativos a un intervalo de confianza del $95 \%(p<0,05)$.

\section{RESULTADOS}

De la cohorte de 137 niños anémicos encontrados en la primera etapa, accedieron a participar 109: 77 niños indígenas y 32 niños no indígenas. Para la segunda etapa, las pérdidas en el seguimiento fueron de $10 / 87(11,5 \%)$ y de18/50 (36\%) de niños indígenas y no indígenas respectivamente. En la tercera etapa debieron ser excluidos los niños que abandonaron la suplementación, quedando un total de 45 niños indígenas y 19 no indígenas, Figura 1.

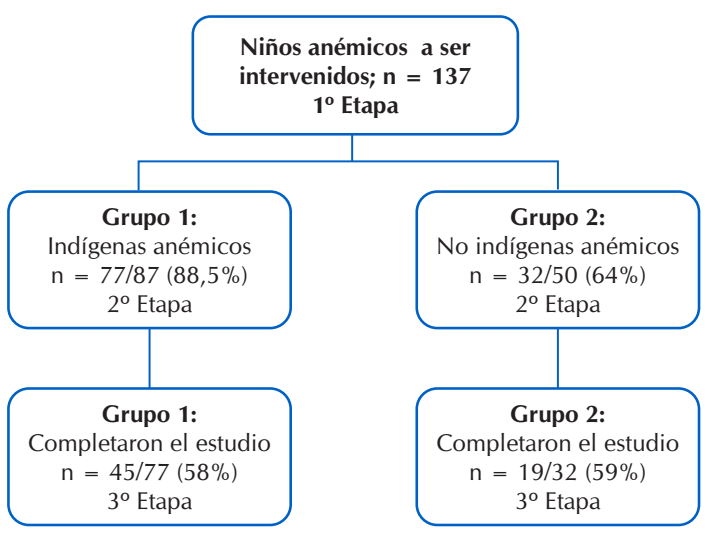

Figura 1. Niños participantes según las etapas del estudio.

En la Tabla 1 se observan indicadores demográficos, nutricionales y coproparasitológicos de la población de estudio.

Tabla 1. Características demográficas, estado nutricional y coproparasitológico de ambas poblaciones estudiadas en la etapa 3 (post intervención) ( $\mathrm{n}=109)$.

\begin{tabular}{|c|c|c|}
\hline Variables & $\begin{array}{l}\text { Población Indígena } \\
\qquad(\mathrm{n}=77)\end{array}$ & $\begin{array}{l}\text { Población No Indígena } \\
\qquad(\mathrm{n}=32)\end{array}$ \\
\hline \multicolumn{3}{|l|}{ Edad (meses) } \\
\hline Media DE & $40,7 \pm 16,2$ & $41,1 \pm 16,3$ \\
\hline \multicolumn{3}{|l|}{ Peso (kg) } \\
\hline Media DE & $13,4 \pm 2,8$ & $15,0 \pm 3,5$ \\
\hline \multicolumn{3}{|l|}{ Talla (cm) } \\
\hline Media DE & $91,2 \pm 9,7$ & $96,4 \pm 11,8$ \\
\hline \multirow{3}{*}{ Sexo } & $\mathrm{N}(\%)$ & $\mathrm{N}(\%)$ \\
\hline & M 43 (56): & $24(75)$ \\
\hline & F: 34 (44) & $8(25)$ \\
\hline Estado nutricional $*$ & $\mathrm{~N}(\%)$ & $\mathrm{N}(\%)$ \\
\hline Desnutrido & $4(5)$ & 0 \\
\hline Riesgo desnutrición & $8(10)$ & $2(6)$ \\
\hline Eutrofico & $49(64)$ & $28(88)$ \\
\hline Sobrepeso/obesidad & $16(21)$ & $2(6)$ \\
\hline Estado & $n=66$ & $\mathrm{n}=30$ \\
\hline coproparasitológico $* *$ & $\mathrm{~N}(\%)$ & $\mathrm{N}(\%)$ \\
\hline Protozooarios & $22(29)$ & $13(41)$ \\
\hline Vermes & $6(8)$ & $1(3)$ \\
\hline Vermes y Protozoarios & $6(8)$ & 0 \\
\hline Sin parasitos & $32(41)$ & $16(50)$ \\
\hline
\end{tabular}

$(*)$ niños de 6 a 23 meses evaluados según P/E; niños de 24 a 59 mese evaluados
$\left({ }^{*}\right)$ solo los participantes en quienes se realizo el estudio coproparasitologico. 
En la tabla 2 se observa el análisis intragrupo, de la tercera etapa del estudio (post intervención) donde se evalúan las diferencias entre los niveles de las medianas de hemoglobina autopareadas antes después de la intervención, encontrándose en los grupos 1 y 2 un aumento significativo de la mediana de hemoglobina después de la intervención, tanto en la población de niños indígenas como en la no indígena de 5,0 g/L y 6,0 g/L de hemoglobina respectivamente.

Tabla 2. Comparación de los niveles de las medianas de hemoglobina intragrupos de la población con anemia $\mathrm{n}=64$.

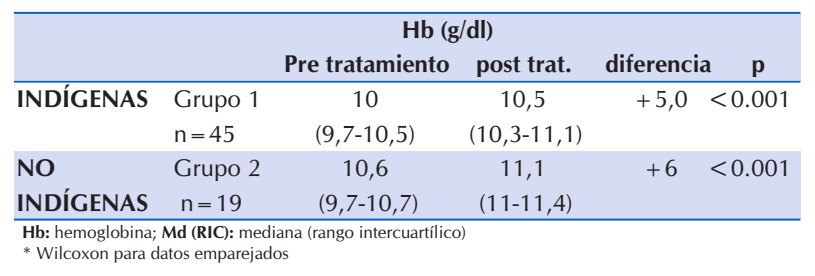

En la primera etapa todos los niños anémicos de ambos grupos presentaron gradaciones de anemia leve (AL) o moderada (AM). En la tercera etapa el $31 \%$ y el $84 \%$ de los niños indígenas y no indígenas dejaron de ser anémicos, Tabla 3.

Tabla 3. Población con anemia y los grados de anemia pre y post tratamiento $=64$.

\begin{tabular}{lllcc}
\hline & \multicolumn{3}{c}{ Grados de anemia } \\
& & Pre tratamiento & $\mathbf{n}(\%)$ & Post tratamiento $\mathbf{n ~ ( \% ) ~}$ \\
\hline INDÍGENAS & $\mathrm{n}=45$ & $\mathrm{~N}(\%)$ & $\mathrm{N}(\%)$ \\
& & Anemia leve & $19(42)$ & $26(58)$ \\
& & Anemia mod. & $26(58)$ & $5(11)$ \\
NO & & Sin anemia & $14(31)$ \\
INDÍGENAS & $\mathrm{n}=19$ & $\mathrm{~N}(\%)$ & $\mathrm{N}(\%)$ \\
& & Anemia leve & $13(68)$ & $2(11)$ \\
& & Anemia mod. & $6(32)$ & $1(0,5)$ \\
& & Sin anemia & $16(84)$ \\
\hline
\end{tabular}

Se observó un consumo adecuado del suplemento en $58 \%$ de los niños indígenas y en $59 \%$ de no indígenas, Figura 1. Los motivos principales del abandono de la suplementación fueron: la falta de accesibilidad al centro de salud para retirar el suplemento por la lejanía de las comunidades y caminos en mal estado $78 \%(n=25 / 32)$ en los indígenas y $61 \%(n=8 / 13)$ en los no indígenas, efectos colaterales del suplemento (nauseas, vómitos, diarrea, estreñimiento) $13 \%(n=4 / 32)$ en los indígenas y $39 \%(n=5 / 13)$ en los no indígenas, dificultad de administración porque el niño no coopera $6 \%(\mathrm{n}=2 / 32)$ en los indígenas, no sabe o no contesta $3 \%(n=1 / 32)$ en los indígenas.

\section{DISCUSIÓN}

El incremento de la hemoglobina demostrada en el análisis intragrupo, en ambas poblaciones estudiadas, creemos probable fue debido, a la intervención con el suplemento con hierro, ya que las condiciones ambientales, sociales y geográficas permanecieron sin cambios en esas comunidades, que presentaron también una baja frecuencia de vermes y más de la mitad de los niños con un estado nutricional adecuado, observándose desnutrición sólo en la población indígena; sin embargo, el tiempo transcurrido entre la medición basal de hemoglobina y la intervención fue una limitante, relacionada a la distancia y el aislamiento en que se encuentran las comunidades estudiadas, la necesidad de recursos humanos, económicos y de logística, tratándose de zonas con camino de tierra muy distantes de los Puestos de Salud.

Si bien es alentador el aumento cuantitativo significativo de la mediana de hemoglobina en los niños indígenas, la misma continuó permaneciendo por debajo del valor de corte, de $110 \mathrm{~g} / \mathrm{L}$, lo que podría evidenciar una recuperación más lenta, debido posiblemente a las frágiles condiciones basales de los niños indígenas tales como, el estado de pobreza en el cual viven los grupos vulnerables del área rural en Paraguay las precarias condiciones de saneamiento y eliminación de desechos, las costumbres y características culturales propias de los nativos paraguayos ${ }^{(19,20)}$, así como la situación de ruralidad $^{(21)}$ y aislamiento, condiciones todas que los hacen más propensos a las infecciones y diarreas.

Con respecto a los grados de anemia se observó una mejoría en los niños indígenas, que en su mayoría pasaron a un estado de anemia leve y $31 \%$ dejaron de ser anémicos; Aguirre y col $^{(22)}$ en el trabajo en niños indígenas de México menores de dos años del área rural, que fueron intervenidos con harina fortificada, encontraron un incremento de la hemoglobina superior en 4 puntos al nuestro, relacionado probablemente con un periodo mayor de tiempo para el monitoreo. 
Es de destacar el papel gravitante que ocuparon los promotores de salud, que pertenecían a sus comunidades indígenas, en la adherencia al tratamiento, teniendo en cuenta que volvieron a participar el 93\% de los niños indígenas (109/117).

En los niños no indígenas también se observó un incremento de la mediana de hemoglobina, superando al valor de corte de $110 \mathrm{~g} / \mathrm{L}$ y más del $80 \%$ dejó de ser anémico; debido probablemente a que los niños se encontraban en mejores condiciones nutricionales y coproparasitológicas, consideramos que el efecto hubiese podido ser aún mayor, sin embargo una de las limitantes del estudio fue la baja adherencia de los niños no indígenas al tratamiento, que fue del $53 \%$ (58/109), relacionado posiblemente a la falta de visibilidad de la anemia como enfermedad, de no contar con un mayor acceso a la información, de educación sobre la enfermedad y sus consecuencias en la primera infancia, debido a que ésta se desarrolla en forma silenciosa, favoreciendo el desconocimiento de su gravedad, y habría incidido para que no asistan a los Puestos de Salud a retirar el suplemento, así como la influencia de factores socioambientales propios de las zonas rurales como ser la necesidad de viajar grandes distancias para recibir atención de salud, los caminos intransitables, o la falta de un cuidador para los hijos pequeños que quedan en el hogar. Aparco y Huamán-Espino resaltan el papel esencial de la madre en el consumo del suplemento, brindarles motivación e información sobre el problema de la anemia, dejando en claro que, para hacer frente a un problema nutricional no se puede organizar un plan de intervención basado sólo en un producto ${ }^{(9)}$.

Urquidi y col, en niños entre 6 meses a dos años, residentes del área urbana en Bolivia, encontraron un incremento de hemoglobina de $11 \mathrm{~g} / \mathrm{L}$, superior en cinco puntos a los $6 \mathrm{~g} / \mathrm{L}$ encontrados en nuestro estudio en niños no indígenas con el suplemento sulfato ferroso, probablemente debido a que nuestro estudio se realizó en el área rural, otro factor a tener en cuenta es la mejor adherencia al tratamiento encontrada por Urquidi y col con otro suplemento (fumarato ferroso) diferente al convencional de sulfato ferroso, que podría favorecer el consumo, ligado a su sabor, ya que el hierro se encuentra encapsulado ${ }^{(15)}$.

Finalmente, creemos que es necesario brindar mayor información a los padres y a la comunidad sobre el problema de la anemia, sus consecuencias, prevención y tratamiento de acuerdo a las características de cada población, de manera que puedan empoderarse. Considerando que la anemia es una afección prevenible, es necesario un abordaje integral, para que la intervención sea beneficiosa y sostenida en el tiempo, y la administración intermitente del suplemento a todos los niños. La información generada en este trabajo pretende contribuir con las autoridades pertinentes a realizar las intervenciones enfocadas a la prevención de la anemia y a la mejoría de la calidad de vida de estas comunidades, de manera que cada niño pueda desplegar sus potencialidades y tener igualdad de oportunidades.

\section{CONCLUSIÓN}

Ambas poblaciones de niños anémicos indígenas y no indígenas presentaron un aumento significativo de los niveles medios de hemoglobina post tratamiento con suplemento con hierro. La mayoría de los no indígenas pasaron al estado no anémico y aunque solo el $30 \%$ de los indígenas dejaron de serlo la mayoría mejoró su grado de anemia.

\section{AGRADECIMIENTOS}

Agradecemos a la Fundación Acción Contra el Hambre (ACF) en la persona de la Sra. Lucía Oliveira, Directora Residente en Paraguay durante el periodo de estudio, y el equipo técnico que en el marco del proyecto "Mejora de la soberanía alimentaria y nutricional en Paraguay" cofinanciado por la Agencia Española de Cooperación Internacional para el Desarrollo (AECID) hizo posible este estudio. Al personal de salud del Departamento de Bioquímica Clínica 


\section{REFERENCIAS BIBLIOGRÁFICAS}

1. Organización Mundial de la Salud. Declaración conjunta de la Organización Mundial de la Salud y el Fondo de las Naciones Unidas para la Infancia: la anemia como centro de atención, hacia un enfoque integrado para un control eficaz de la anemia. Ginebra: OMS; 2004.

2. Walker SP, Wachs TD, Grantham-McGregor S, Black MM, Nelson CA, Huffman SL, et al. Inequality in Early Childhood: Riskand Protective Factors for Early Child Development. Lancet. 2011;378(9799):1325-38.

3. Alcázar L. Impacto económico de la anemia en el Perú [Internet]. Lima: GRADE; Acción contra el Hambre; 2012. [Citado 27 de julio de 2018] Disponible en: http://www.grade.org.pe/upload/publicaciones/archivo/ download/pubs/LIBROGRADE_ANEMIA.pdf

4. Bruno S, Osorio A. La pobreza infantil multidimensional en Paraguay [Internet]. Documento de trabajo. Paraguay: UNICEF; 2015. [Citado 9 de julio de 2019] Disponible en: https://www.unicef.org/paraguay/spanish/unicefpypobrezamultidimensional.pdf

5. Organización de las Naciones Unidas para la Alimentación y la Agricultura (FAO). Pobreza rural y políticas públicas en América Latina y el Caribe [Internet]. Tomo I. Santiago, Chile: FAO; 2013. [Citado 8 de agosto de 2018]. Disponible en: http://fao.org/alc/u/y2

6. Gillespie S, McLachlan M, Shrimpton R. Combating malnutrition: time to act (English). Health, Nutrition and Population (HNP) discussion paper. Washington DC: World Bank Group; 2003.

7. Mora JO. Iron supplementation: overcoming technical and practical barriers [Internet]. J Nutr. 2002; 132(4 Suppl):853S-5S. [Citado 28 de julio de 2018]. Disponible en:https://www.ncbi.nlm.nih.gov/pubmed/11925496

8. Zavaleta N. Anemia infantil: Retos y oportunidades al 2021.Rev Peru Med Exp Salud Publica. 2017;34(4):588-89.

9. Aparco JP, Huamán-Espino L. Recomendaciones para intervenciones con suplementos de hierro: lecciones aprendidas en un ensayo comunitario en cuatro regiones del Perú. Rev Peru Med Exp Salud Publica. 2017;34(4):709-15.

10. Programa Alimentario Nutricional Integral, Unidad Técnica del Gabinete Social, Presidencia de la República del Paraguay. Evaluación de consistencia y resultado [Internet]. Asunción; 2017. [Citado 27 de julio de 2018] Disponible en https://www.gabinetesocial.gov.py/ar chivos/documentos/PANI-docu-eva_uhfqt18i.pdf

11. Nuñez N, Sanabria M, Aguilar G. Eficacia de la intervención nutricional del Programa Alimentario Nutricional Integral (PANI) en niños beneficiarios que asisten al Hospital Regional de Concepción. Pediatr. (Asunción). 2015; Supl(42):16

12. World Health Organization, United Nations Children's Fund, United Nations University, (WHO/UNICEF/UNU). Iron deficiency anaemia: assessment, prevention and control: a guide for programme managers. Ginebra: OMS; 2001.

13. Echagüe G, Sosa L, Díaz V, Funes P, Ruíz I, Pistilli N, et al. Anemia en niños indígenas y no indígenas menores de 5 años de comunidades rurales del Departamento de Caazapá. Pediatr. (Asunción). 2013;40(1):19-28.

14. Instituto Nacional de Alimentación y Nutrición. Protocolo de implementación del Programa Alimentario Nutricional Integral para profesionales de salud [Internet]. Paraguay; 2011. [Citado 15 de febrero de 2013] Disponible en: http://www.inan.gov.py/docs/protocolo_pani

15. Urquidi C, Mejía H, Vera C. Adherencia al tratamiento de la anemia con fumarato ferroso microencapsulado. Rev Soc Bol Ped. 2007; 46(1):3-11.

16. Hulley S, Cummings S, Browner W, Grady D, Newman T. Diseño de investigaciones clínicas. 3 ed. Barcelona, España: Lippincott Williams \&Wilkins; 2007.

17. Organización Mundial de la Salud. Concentraciones de hemoglobina para diagnosticar la anemia y evaluar su gravedad [Internet]. Ginebra: OMS; 2011. [Citado 19 de junio de 2018] Disponible en: http://www. who.int/vmnis/indicators/haemoglobin_es.pdf

18. World Health Organization. WHO Child Growth Standards: Methods and development. Geneva:WHO; 2006.

19. Echagüe G, Sosa L, Díaz V, Funes P, Rivas L, Granado $D$, et al. Malnutrición en niños menores de 5 años indígenas y no indígenas de zonas rurales, Paraguay. Mem. Inst. Investig. Cienc. Salud. 2016; 14(2):25-34.

20. Echagüe G, Sosa L, Díaz V, Ruiz I, Rivas L, Granado D, et al. Enteroparasitosis en niños bajo 5 años de edad, indígenas y no indígenas, de comunidades rurales del Paraguay. Rev Chilena Infectol. 2015;32(6):649-657.

21. Oliveira CS, Cardoso MA, Araújo TS, Muniz PT. Anemia en criancas de 6 a 59 meses e factores asociados no Municipio de Jordao; Estado do acre, Brasil. Cad. Saúde Pública 2011;27:1008-20.

22. Aguirre J, Chávez A, Medina B, García E, Carrasco M, Guarneros N. Impacto del suministro de harina de maíz fortificada en la anemia de preescolares de zonas indígenas de México. Gac Sanit. 2013; 27(6):541-544. 